\title{
Integrating Fingerprint Verification into the Smart Card-Based Healthcare Information System
}

\author{
Daesung Moon, ${ }^{1}$ Yongwha Chung, ${ }^{2}$ Sung Bum Pan, ${ }^{3}$ and Jin-Won Park ${ }^{4}$ \\ ${ }^{1}$ Biometrics Technology Research Team, ETRI, Daejeon 305-700, South Korea \\ ${ }^{2}$ Department of Computer and Information Science, Korea University, Jochiwon, Chungnam 339-700, South Korea \\ ${ }^{3}$ Department of Information Control and Instrumentation Engineering, Chosun University, Gwangju 501-759, South Korea \\ ${ }^{4}$ School of Games, Hongik University, Jochiwon, ChungNam 339-701, South Korea \\ Correspondence should be addressed to Yongwha Chung, ychungy@korea.ac.kr
}

Received 10 October 2008; Revised 13 May 2009; Accepted 14 September 2009

Recommended by Stephanie Schuckers

\begin{abstract}
As VLSI technology has been improved, a smart card employing 32-bit processors has been released, and more personal information such as medical, financial data can be stored in the card. Thus, it becomes important to protect personal information stored in the card. Verification of the card holder's identity using a fingerprint has advantages over the present practices of Personal Identification Numbers (PINs) and passwords. However, the computational workload of fingerprint verification is much heavier than that of the typical PIN-based solution. In this paper, we consider three strategies to implement fingerprint verification in a smart card environment and how to distribute the modules of fingerprint verification between the smart card and the card reader. We first evaluate the number of instructions of each step of a typical fingerprint verification algorithm, and estimate the execution time of several cryptographic algorithms to guarantee the security/privacy of the fingerprint data transmitted in the smart card with the client-server environment. Based on the evaluation results, we analyze each scenario with respect to the security level and the real-time execution requirements in order to implement fingerprint verification in the smart card with the client-server environment.
\end{abstract}

Copyright () 2009 Daesung Moon et al. This is an open access article distributed under the Creative Commons Attribution License, which permits unrestricted use, distribution, and reproduction in any medium, provided the original work is properly cited.

\section{Introduction}

A smart card is a credit-card-sized plastic card with an embedded chip that can be memory or may include a microprocessor [1]. A microprocessor chip in a smart card can add, delete, or manipulate information in its memory and hence offer complex data security functionalities. These smart cards have been used in many applications such as health cards, e-passport, and e-ID cards for more than 10 years [2-4]. In this paper, we describe an example where a smart card is applied in the healthcare information system. One of the most popular smart card solutions is Gemalto Cryptoflex JavaCard [5], equipped with a 16-bit microcontroller (Infineon SLE66CX322P, compatible with standard SAB 8051 processor) and an additional crypto processor for RSA and DES computations. The card has ROM, EEPROM, and RAM. A Java Applet implements the Chip Authentication Program.
The usual way of obtaining relevant patient data is connecting to the hospital database. However, we may experience the situation where no network connection facility (e.g., ambulance) is available. Also, hospitals cannot open the patient data stored in the hospital database via Internet because of the security/privacy of the patient and/or different network environments. These problems can be solved by using portable storage devices such as smart cards. That is, a doctor may take patient information from the smart card at the time of consultation.

Because of the advantage of the smart card, using the smart card in healthcare becomes popular in such countries as France, Germany, and Taiwan. Also, several solutions are already implemented [5-10]. For example, Taiwan was the first country introducing the nationwide smart card-based healthcare information system in 2003. Over 22 million patient health cards have been issued, as well as over 345000 health professional cards giving the doctor access to medical 
information. In EU, several projects about smart cardbased healthcare service have been performed such as Sesam Vitale in France, DENTcard in Germany [7], Transcards [8], NETLINK [9], and NETC@RDS project [10].

Deployed as of 1998, SESAM-Vitale currently links more than 223000 healthcare professionals with the Health Insurance System, for the benefit of millions of insured persons who have the Vitale card. The NETC@RDS initiative is devoted to establish new improved healthcare administration services for mobile citizens across EU. The actual phase aims at deploying e-health services via the European Health Insurance Card through a wide trans-European network simplifying healthcare access procedures. NETC@RDS successfully tested the electronic version of the European Health Insurance Card (EHIC) during its first and second Market Validation phases in 85 pilot sites of the $10 \mathrm{EU}$ member states. The Initial Deployment phase of the project-launched on June 1, 2007-will deploy operational services in all targeted sites, to total of 305 service sites serving 566 service points across the 15 participating countries.

NETLINK is a project of the fourth framework programme on research and development of European commission HC 4016. Countries involved in the NETLINK consortium (France, Germany, Italy, and the Province of Québec) have set up or are in the process of setting up new nationwide information systems in the healthcare sector based on the use of modern technologies: smart cards (used by health professionals and patients), computers (used by health professionals, hospitals, health Insurance funds), large networks, trusted third parties (for security purpose).

In the security and the privacy terms, several issues should be considered in developing the smart card-based healthcare information system; the ways to certify different devices (card, card reader, terminal), the methods to authenticate users (health professionals and patients), and the amount of information about a patient to be stored in the smart card, for example, patient personal information and emergency contact information. There are many other issues to consider and the details of other issues can be found in $[11,12]$. In this paper, we consider the methods to authenticate the cardholder, especially patients in a largescale hospital database.

In general, the visible information on the health card may contain the cardholder's name, identification number, birth date, photo, and the card serial number. The contents inside the card can be divided into four segments: basic data, health insurance data, medical data, and public health administration data $[13,14]$. The basic data segment stores the identification information for both the cardholder and the card itself. The contact person information in case of emergency and the cryptographic key for security can also be stored. The health insurance data segment is comprised of the cardholder's insurance information and service data for insurance claims. The medical data segment contains the information for important physician orders, prescriptions, and drug allergies. With the advance in the smart card technology, more medical information can be stored in the card. Finally, the public health administration data segment is used for recording personal data pertaining to public health such as vaccination records and organ donation notes. Additionally, a separate smart card can be used as an identification card for a medical staff.

As more information is stored in the smart card, it becomes important to protect the information it contains. However, the current card holder verification method in the healthcare services is based on the password/PIN such as SESAME-VITALE. Most of people set their passwords/PINs based on words or numbers that they can easily remember. Thus, the passwords are easy to crack by guessing or a simple brute force dictionary attack. Although it is possible and even advisable to keep different passwords for different applications, most of people use the same password across different applications. If a single password is compromised, it may open many doors. "Long and random" passwords are more secure but harder to remember, which prompts some users to write them down in accessible locations. Such passwords also result in more system help desk calls for being forgotten or expired. Cryptographic techniques such as PKI $[15,16]$ can provide very long passwords that need not to be remembered but are in turn protected by simple passwords, thus defeating their original purpose.

In recent years, there is an increasing trend of using biometrics, which refers to the personal biological or behavioral characteristics used for verification or identification $[17,18]$. It relies on "something that you are," and can inherently differentiate between a verified person and a fraudulent imposter. The problem of resolving the identity of a person can be categorized into two distinct types, verification and identification. Verification matches a person's claimed identity to his/her previously enrolled pattern (i.e., "oneto-one" comparison). However, identification identifies a person from the entire enrolled population by searching a database for match (i.e., "one-to-many" comparison). In this paper, we focus on fingerprint because it is relatively mature and its scanning unit is cheaper than other biometrics such as iris [18]. Also, we will focus on verification only because we assume each patient or medical staff holds his/her smart card. (Note that, however, fingerprints can also be applied to healthcare services without the smart card.)

Since fingerprints cannot be lost or forgotten like passwords, fingerprints have the potential to offer higher security and more convenience for user authentication [18]. For example, fingerprints are significantly more difficult to copy, share, and distribute than passwords. That is, the main advantage of a fingerprint recognition solution is the convenience while maintaining sufficiently high security. In general, security with large data is regarded as higher than that with small data although this does depend greatly on the way it is implemented. Fingerprint data size is typically $70 \mathrm{~KB}$ for images and $500 \mathrm{~B}$ for features, much larger than that of the password with 10B. Furthermore, large fingerprint data need not to be memorized. Especially in healthcare services, fingerprints have additional advantage over the password. The emergency data set such as his/her blood type and contact person information can be accessed from the smart card by using his/her finger in the emergency medical situation when the patient is unconscious. 
However, fingerprint-based recognition has some disadvantages as well $[19,20]$. A compromised password can be canceled and a new password can be issued as often as desired, whereas people have only 10 fingers. If a fingerprint is compromised repeatedly, it cannot be replaced eventually [18]. Finally, in principle, a fingerprint template stolen from one application may be used in another application. These issues are especially important in pervasive computing where the fingerprint data must be carefully protected because of privacy concerns. Also, we need more computation to authenticate users with the fingerprint data than the password.

In large-scale healthcare services, the computational overhead caused by the deployment of the fingerprint as well as the protection of the fingerprint data should be considered. However, only limited research has been carried out in this direction $[21,22]$. In this paper, we consider the possible scenarios to integrate fingerprints into smart cards and evaluate each scenario in terms of security, privacy, and computational overhead (i.e., cost of the smart card). Also, for the cheapest scenario (i.e., the fingerprint data is transmitted to a remote server for verification), the collective performance of fingerprint verification and the authentication protocol on the client-server model is analyzed.

The rest of the paper is structured as follows. Section 2 explains the overview of typical fingerprint verification and three strategies for integrating fingerprints into the smart card. Sections 3 and 4 describe the fingerprint verification scenarios for the fingerprint smart card and the client-server environments, respectively. The results of the performance evaluation are described in Section 5, and the conclusions are given in Section 6.

\section{Background}

2.1. Biometric-Based User Authentication for Healthcare. The healthcare industry is confronted with solving the legal requirement to protect medical information of patients. Medical information is available in the computer network, thus could be used illegally. Laws call for the protection of patient privacy and also for standardization of medical data. Biometrics are expected to be chosen as possible means for user authentication for healthcare, since biometrics provide a secure method for patient identification and extracting personal data for treatment. In a large hospital, there may occur the following three types of healthcare services that are related to biometric information.

Access to Personal Medical Information. The largest part of biometric usage in healthcare industry may be enhancing individuals' access to their personal information. It seems likely that patients will demand access to their information, while demanding that such information be kept secure. Medical information may be stored on smart cards or on networks; in either case, the biometric is a gateway to personal information.
Emergency Patient Identification. In emergency medical situations, correct and immediate patient authentication is critical. For individuals without identification and unable to communicate, biometric information provides a unique form of authentication. Developing this type of biometric identification system includes enrolling a sufficient number of users to achieve critical mass and the availability of biometric devices in emergency situations and locations. In this paper, we assume each patient holds his/her smart card and the emergency dataset such as his/her blood type and contact person information can be accessed from the smart card by using his/her finger in emergency medical situations.

2.2. Fingerprint Verification. The fingerprint is chosen for verification and for identification in this paper. It is more mature in terms of the algorithm availability and feasibility. Fingerprint verification and identification algorithms can be classified into two categories: image-based and minutiaebased $[17,18]$.

A minutiae-based fingerprint verification system has two phases: enrollment and verification. In the off-line enrollment phase, an enrolling fingerprint image for each user is processed, and the features called minutiae are extracted and stored in a server. In the online verification phase, the minutiae extracted from an input image is compared to the stored template, and the result of the comparison is returned.

In general, there are six logical modules involved in the fingerprint verification system [18]: Fingerprint Acquisition module, Feature Extraction module, Matching module, Storage module, Decision module, and Transmission module.

The Fingerprint Acquisition module contains an input device or a sensor that captures the fingerprint information from the user. It first refines the fingerprint image against the image distortion obtained from the fingerprint sensor. A typical process consists of three stages. The binary conversion stage applies a lowpass filter to smooth the high frequency regions of the image and threshold to each subsegment of the image. The thinning operation generates a onepixel-width skeleton image by considering each pixel with its neighbors. In the positioning operation, the skeleton obtained is transformed and/or is rotated such that valid minutiae information can be extracted.

The Feature Extraction module refers to the extraction of features in the fingerprint image. After this step, some of the minutiae are detected and stored into a pattern file, which includes the position, the orientation, and the type (ridge ending or bifurcation) of the minutiae.

Based on the minutiae, the input fingerprint is compared with the enrolled fingerprint retrieved from the Storage module. Actually, the Matching module is composed of the alignment operation and the matching operation. In order to match two fingerprints captured with unknown direction and position, the differences of direction and position between two fingerprints are detected, and the alignment between them needs to be accomplished. Therefore, in this alignment operation, transformations such as translation and rotation between two fingerprints are estimated, and two minutiae are aligned according to the estimated parameters. 
If the alignment is performed accurately, the following matching operation can be regarded as a simple point pattern matching. In the matching operation, two minutiae are compared based on their position, orientation, and type. Then, a matching score is computed. The Decision module receives the score from the matching module and, using a confidence value based on the security risks and the risk policy, interprets the result of the score, thus reaching a verification decision. The Transmission module provides the system with the ability to exchange information between all other modules.

\subsection{Integrating Fingerprint into the Smart Card. Fingerprint} technologies have been proposed to strengthen the verification mechanisms in general by matching a stored fingerprint template to a live fingerprint features $[17,18]$. In the case of verification using a smart card, intuition suggests that the match should be performed by the smart card. However, this is not always possible because of the complexity of the fingerprint information, and because of the limited computational resources available to current smart cards. In general, three strategies of fingerprint verification can be identified as follows [23-26].

Store-on-Card. The fingerprint template is stored on a smart card. It must be retrieved and transmitted to a card reader that matches it to the live template acquired from the user by the fingerprint sensor. Cheap memory cards with no or small operating systems are generally sufficient for this purpose.

Match-on-Card. The fingerprint template is stored on a smart card, which also performs the matching with the live template. Therefore, a microprocessor on the smart card is necessary. The smart card must contain an operating system running a suitable match application. It is not possible to steal information stored in the card since a successful match enables the use of the certificates on the card without the need of stored PINs or passwords. Even in the unlikely event that a card is tampered with; only limited damage is caused since only that specific user's credentials are hacked. An attack on multiple users means that the attacker must get hold of all users' cards. In this strategy, the templates are never exposed to a nontamper proof environment and the user carries his/her own templates.

System-on-Card. This is a combination of the two previous strategies. The fingerprint template is stored on a smart card, which also performs the matching with the live template, and includes the fingerprint sensor to acquire, select, and process the live template. This strategy is the best in terms of the security as everything takes place on the smart card. Embedding a fingerprint acquisition on a smart card provides all the privacy and security solutions but, unfortunately, it is expensive and presents more than one realization problem.

The benefits derived from the Match-on-Card are valuable in themselves: using its own processing capabilities, the smart card decides if the live template matches the stored template closely enough to grant the access to its private data. Nevertheless this scheme presents a danger: we have no certainty that a fingerprint acquisition has been collected through live-scan and there is the risk of an attacker's sniffing the fingerprint data and later using it to unlock the card in a replay attack.

\section{Fingerprint Verification Scenarios for the Smart Card-Reader Model}

First, simplifying the scenarios between the smart card and the card reader considered, we assume that the symmetric and/or asymmetric keys are distributed to the smart card and the card reader when the system is installed and no further key exchange is required.

We explained three strategies for integrating the fingerprints into the smart card in the previous section. In this section, we consider five different scenarios [20-26, 28, 29]. As shown in Figure 1, SCENARIO 1 and SCENARIO 2 are Store-on-Card strategies because the smart card stores the fingerprint template. Also, as shown in Figure 2, SCENARIO 3 and SCENARIO 4 are Match-on-Card strategies because the matching module takes place on the smart card. Finally, SCENARIO 5 is the System-on-Card strategy (Figure 3). Within the Store-on-Card and the Match-on-Card scenarios, we can differentiate those in which the fingerprint sensor is built into the smart card (SCENARIO 2 and SCENARIO 4) and those where it is in the card reader (SCENARIO 1 and SCENARIO 3).

Store-on-Card: SCENARIO 1 and SCENARIO 2. In SCENARIO 1, the fingerprint sensor is built into the card reader. The user template is transferred from the card to the reader. The reader takes the fingerprint image provided by its builtin fingerprint sensor, performs the feature extraction, and also matches the features to the template provided by the card. The reader then informs the card whether verification has been successful or not.

On the other hand, the fingerprint sensor in SCENARIO 2 is built into the card. The fingerprint image and the user template are transferred from the card to the reader. The reader performs feature extraction and matches the features to the template. The reader then informs the card whether verification has been successful or not.

Match-on-Card: SCENARIO 3 and SCENARIO 4. In SCENARIO 3, the fingerprint sensor is built into the card reader. The reader takes the image provided by the builtin fingerprint sensor and performs feature extraction. The extracted features are sent to the card, which then performs the matching module and reaches the verification decision module.

The fingerprint sensor in SCENARIO 4 is built into the card. The fingerprint image is transferred from the card to the reader. The reader performs the feature extraction module only, and transfers the extracted features back to the card. The card then performs the matching module. 
Scenario 1

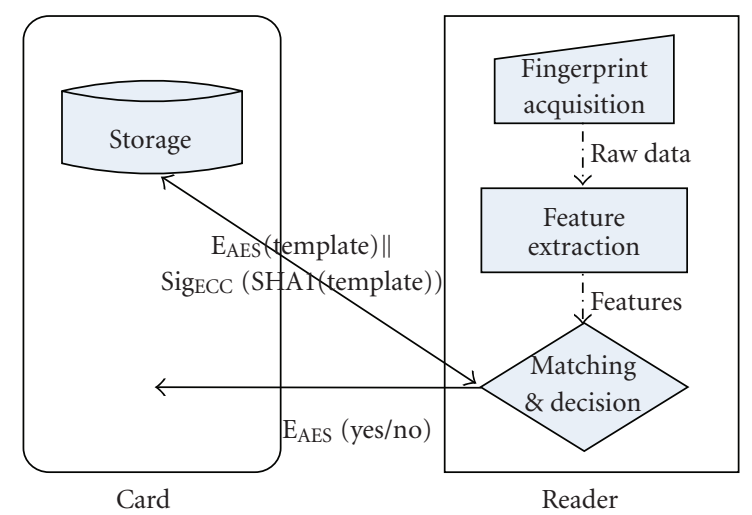

Scenario 2

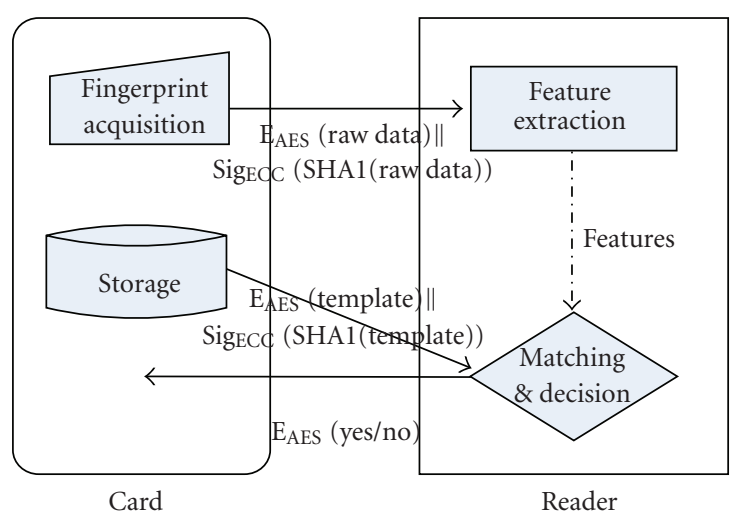

FIGURE 1: Illustration of the integrating scenarios [23-26] for store-on-card and the corresponding X9.84 [27] implementations.

Scenario 3

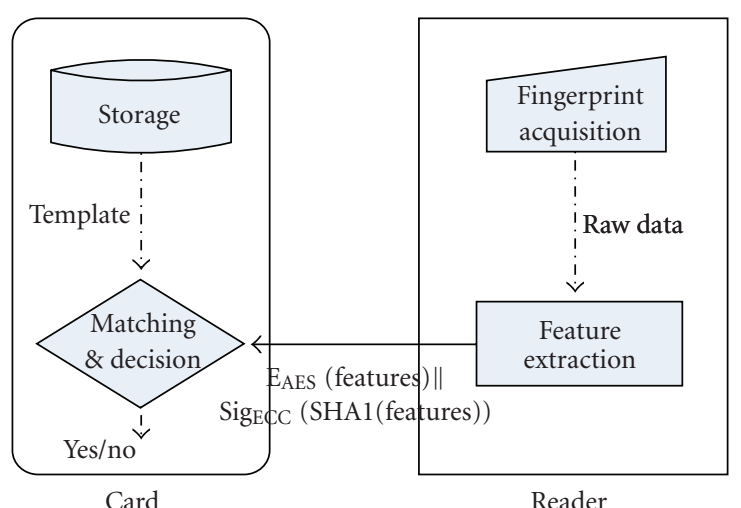

Scenario 4

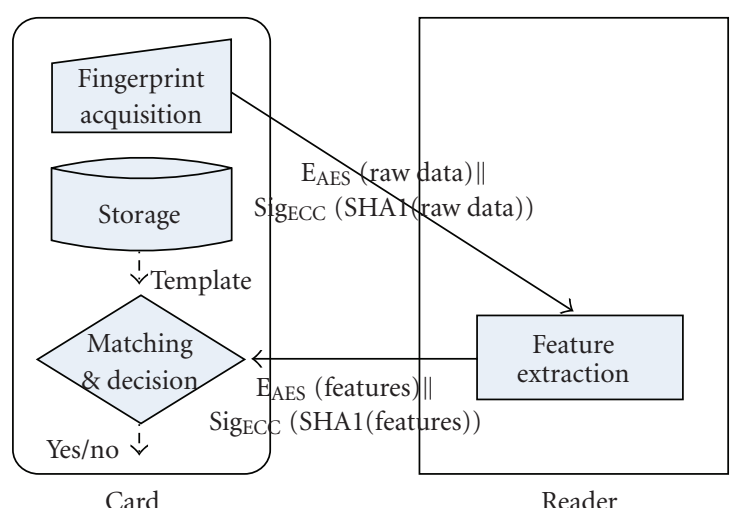

FIGURE 2: Illustration of the integrating scenarios [23-26] for match-on-card and the corresponding X9.84 [27] implementations.

Scenario 5

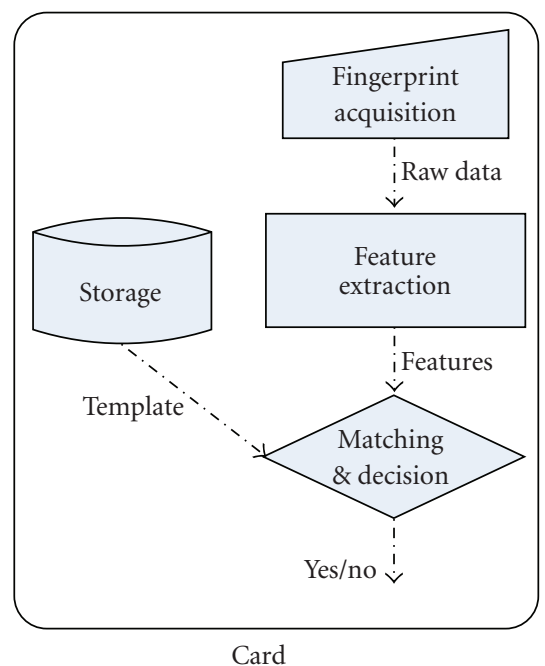

Card

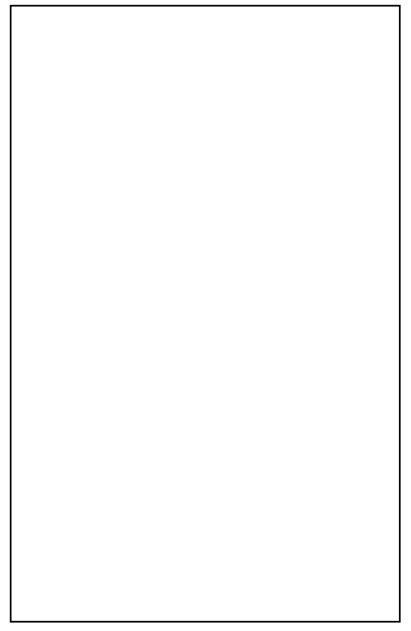

Reader

FIGURE 3: Illustration of the integrating scenarios [23-26] for system-on-card and the corresponding X9.84 [27] implementations. 
System-on-Card: SCENARIO 5. SCENARIO 5 is System-onCard: that is, all fingerprint verification modules take place on the card.

\section{Fingerprint Verification Scenarios for the Large-Scale Client-Server Model}

As we explained in Section 1, we also consider the clientserver model for remote user authentication using fingerprint. Especially, we consider the healthcare information system using the cheapest fingerprint-based smart cards, that is, SCENARIO 1. In spite of guaranteeing higher security level than SCENARIO 1, other scenarios may not be right choices for large-scale applications such as national healthcare services or large hospitals having millions of patients due to the high implementation cost.

The client-server model for remote user authentication using the SCENARIO 1-based fingerprint card must guarantee the security/privacy as well as the real-time execution requirements. To satisfy those requirements, we first consider possible scenarios for remote fingerprint verification in terms of assigning the tasks of the fingerprint verification to each entity (i.e., client and server). Then, we evaluate the performance of each scenario.

Note that, to provide higher security level in the remote healthcare service, we assume the three-way verification method among the smart card fingerprint data, the live fingerprint data and the fingerprint data stored in the central DB. Also, we denote the three possible fingerprint verification scenarios for the client-server model as SCENARIO 1_1, SCENARIO 1_2, and SCENARIO 1_3, respectively.

Following assumptions are made to simplify the explanation.

(1) Between the client and the server, the same master key is shared when the system is installed.

(2) Entity authentication is completed using proper methods such as trusted Certificate Authority (CA).

(3) The user authentication service is assumed to be requested by the client at which the board control investigator is working.

(4) The execution time to perform some cryptography mechanisms to protect the fingerprint features stored in the smart card and in the server's database is not considered because this research focuses only on the protection of the fingerprint data transmitted.

In fact, these assumptions are reasonable, since the master key sharing operation (described in Assumption 1) needs to be executed only once, and the time for protecting the stored fingerprint data (described in Assumption 4) is negligible. For the purpose of explanation, we define first the following notations:

$N$ : a nonce generated randomly in the client and used as a "challenge;"

$K_{m}$ : a master key shared by both the sensor and the client;
$f(N)$ : a simple function to generate a "response" for $N$;

$K_{s}$ : a shared session key generated for each transmission;

C_Fe: a fingerprint feature stored in the biometric health card;

L_Fe: a fingerprint feature extracted from the live fingerprint image;

S_Fe: a fingerprint feature stored in the DB;

L_Fi: a live fingerprint image;

Mat_CL: a matching result between $C_{-} F e$ and $L \_F e$;

Mat_CS: a matching result between $C \_F e$ and S_Fe.

SCENARIO 1_1, Store-on-Card/the Server Does Everything. In SCENARIO 1_1 as shown in Figure 4, the sensor attached to the client captures a live fingerprint image, and the client extracts some features from the image. Then, the client sends $\mathrm{L} F e$ and $\mathrm{CF} F e$ to the server after applying the encryption and digital signature with the same key received from the server.

After verifying the signature for $\mathrm{LFe}_{-}$and $\mathrm{C}_{-} \mathrm{Fe}$ and decrypting these fingerprint features, the server performs two comparisons with $C_{-F e}-S_{-} F e$ and $C_{-F e}-L_{-} F e$. After checking the two matching results, the server returns a final result to the client. Note that this is a typical scenario of assigning the fingerprint verification tasks to the client-server model and requires five sets of communications for data transmission. This scenario can improve the security level of the fingerprint authentication system because the server can be more secure than the client. A server should be protected by the security experts, while a client maintained by an individual user may be more vulnerable to several attacks such as Trojan Horse [12-14]. On the other hand, the computational workload of the server in this scenario increases as the number of clients increases.

SCENARIO 1_2, Store-on-Card/Extraction by the Client and Matching by the Server. Unlike SCENARIO 1_1, in SCENARIO 1_2 as shown in Figure 5, the comparison with $\mathrm{CFe}-$ $L_{-} F e$ and $\mathrm{C}_{-} \mathrm{Fe}-\mathrm{S}_{-} \mathrm{Fe}$ is executed in the client and the server, respectively. In this scenario, the client sends only $\mathrm{C} F \mathrm{Fe}$ to the server and calculates the matching score between Mat_CS received from the server and Mat_CL resulted in the client.

SCENARIO 1_3, Store-on-Card/The Client Does Everything. In SCENARIO 1_3 as shown in Figure 6, all the tasks except fingerprint acquisition are executed in the client. After encrypting the fingerprint features of the requested user stored in the server's database, the server only transmits it to the client. Thus, this scenario can reduce the workload of the server significantly by distributing the fingerprint authentication tasks into the clients. However, the security level of the fingerprint authentication system can be degraded because the client, which is more vulnerable to several attacks than the server, executes most of the tasks and the system depends on the security of keys stored in the client. 


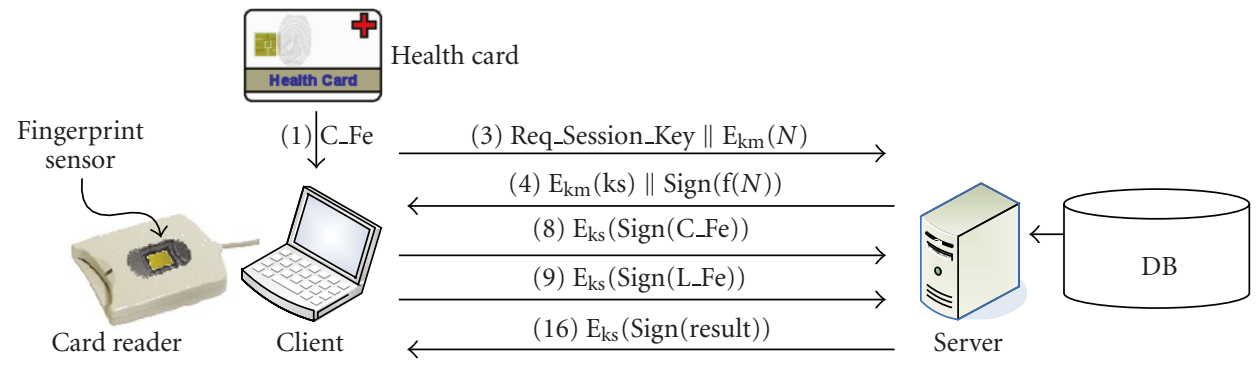

(2) Input live fingerprint of patient

(5) Encrypt and generate signature about $\mathrm{C}_{-} \mathrm{Fe}$

(11) Decrypt and verify signature about L_Fe

(6) Feature extraction about L_Fi

(12) Matching between L_Fe and C_Fe

(7) Encrypt and generate signature about L_Fe

(13) Matching between S_Fe and C_Fe

(10) Decrypt and verify signature about C_Fe

(14) Fusion between Mat_CS and Mat_CL

(15) Encrypt and generate signature about result

FIgURE 4: Illustration of SCENARIO 1_1.

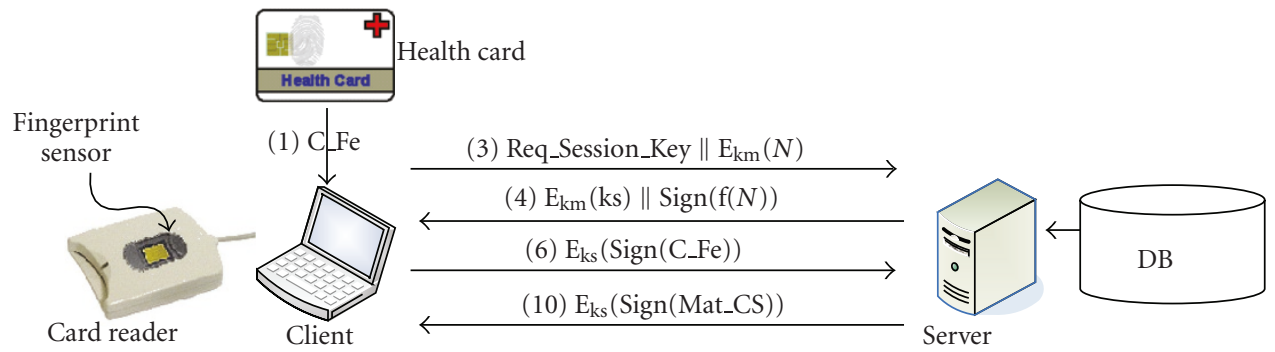

(2) Input live fingerprint of patient

(5) Encrypt and generate signature about C_Fe

(11) Feature extraction about L_Fi

(12) Matching between C_Fe and L_Fe

(13) Decrypt and verify signature about Mat_CS
(14) Fusion between Mat_CS and Mat_CL

(7) Decrypt and verify signature about C_Fe

(8) Matching between C_Fe and S_Fe

(9) Encrypt and generate signature about Mat_CS

FIGURE 5: Illustration of SCENARIO 1_2.

TABLE 1: Number of instructions required for fingerprint verification [25].

\begin{tabular}{lc}
\hline & Number of Instructions \\
\hline Feature Extraction & 451739250 \\
Feature Matching & 21164578 \\
\hline
\end{tabular}

TABLE 2: Number of instructions and estimated time for fingerprint verification.

\begin{tabular}{lcc}
\hline Step & $\begin{array}{c}\text { Estimated time on } \\
\text { ARM7TDMI }\end{array}$ & $\begin{array}{c}\text { Estimated time on } \\
8051\end{array}$ \\
\hline Feature Extraction & 7.5 seconds & 195 seconds \\
Feature Matching & 0.3 seconds & 7.8 seconds \\
\hline
\end{tabular}

\section{Performance Evaluation}

5.1. Evaluation of Fingerprint Verification and the Cryptographic Modules. A fingerprint-based smart card system for user verification must guarantee the user's privacy and provide sufficient authentication for access to patient data as well as the real-time execution requirements. To satisfy the requirements, we first evaluate the logical modules involved in the fingerprint verification system and the cryptographic modules for guaranteeing the integrity and confidentiality of the sensitive fingerprint data transmitted between the smart card and the card reader (see Section 3).

For secure transmission of the fingerprint data, we consider ANSI X.9.84, which is the security standard for fingerprint systems. The ANSI X.9.84 Fingerprint Information Management and Security standard [22] covers the requirements for managing and securing biometric data (fingerprint, iris, voiceprint, etc.) for customer identification and employee verification, mainly in the financial industry. In addition, this standard identifies the digital signature and encryption to provide both integrity and privacy of the fingerprint data. Specifically, 128-bit Advanced Encryption Standard (AES) and Elliptic Curve Digital Signature Algorithm (EDCSA) [8,9] are considered as our symmetric encryption algorithm and digital signature and hash algorithm, respectively (see Figures 1-3). ECDSA is the elliptic curve analogue of the Digital Signature Algorithm (DSA). It is the most widely standardized elliptic curve-based signature scheme, appearing in ANSI X9.62, FIPS 186-2, IEEE 1363-2000, and the ISO/IEC 15946-2 standards as well as several draft standards. Because the most time consuming operations of ECDSA are ECC and the hash operation, we 


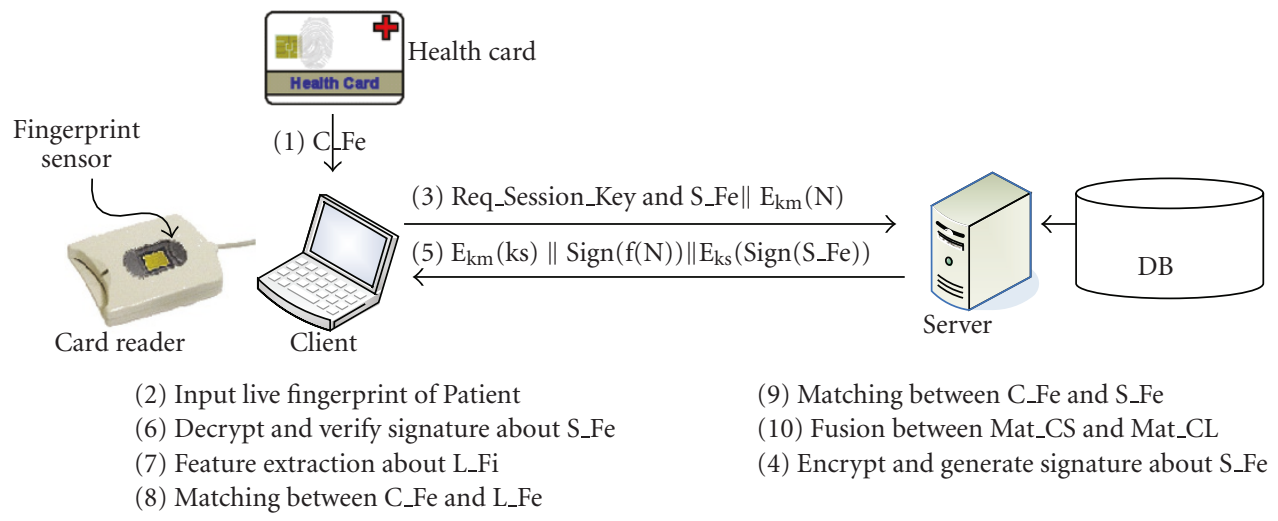

Figure 6: Illustration of SCENARIO 1_3.

TABLE 3: Number of Instructions and Cycles Required for AES.

\begin{tabular}{lccccc}
\hline & & Encryption & AES (128-bit) & \multicolumn{2}{c}{ Decryption } \\
& & No. of instructions & No. of cycles & No. of instructions & No. of cycles \\
\hline \multirow{2}{*}{ ARM7TDMI } & $140 \mathrm{~KB}$ & 292889168 & 485763071 & 406011432 & 690034743 \\
& $1 \mathrm{~KB}$ & 2131620 & 3535199 & 2952268 & 5017575 \\
\hline
\end{tabular}

confine our evaluation to them. Here, SHA1 is used as the hash algorithm.

Table 1 shows the number of instructions of each task in fingerprint verification measured on an instruction simulator, SimpleScalar [30]. Based on Table 1, we can compute the estimated execution time of each task on each processor. Finally, the time to acquire a fingerprint image through the fingerprint sensor is assumed to be about 1 second. Note that the feature extraction module requires a lot of integer operations for image processing, and the computational workload of this module occupies $96 \%$ of the total workload of fingerprint verification.

In order to show the performance requirement of the incard processor, the number of instructions and the estimated execution time on the 8-bit Intel-8051 and 32-bit ARM7based smart cards are summarized in Table 2. According to Table 2, it is impossible to assign the feature extraction or the matching step as well as the preprocessing to the 8051 chip. This is because the computation using the fingerprint data requires a large amount of memory and time. Thus, we adopt ARM7 to realize the Match-on-Card [25], which shows an improved result. Actually, the 32-bit smart card is somewhat expensive to be applied for the ordinary system. Nevertheless, it can be a good solution for the system that should guarantee very high level of security such as in E-Health, E-Business, and E-Government.

Because of the limited processing power of the in-card processor, all of the three steps above cannot be assigned to the in-card processor. Instead, we consider assigning only the third step, matching, to the in-card processor. This is because the first two steps involve rigid image processing computation, which is too exhaustive to be executed in the in-card processor. These computation steps can be easily carried out in real-time by a fingerprint capture device or a card reader equipped with at least a 500 MIPS processor. Therefore, all of the computational steps can be performed in real-time, and the smart card can encapsulate the fingerprint data and perform the comparison securely inside the card.

Also, Table 3 and 4 show the number of instructions of the cryptography modules measured on the simulator ARMulator [31]. The cryptographic modules need to guarantee the privacy of the fingerprint data transmitted between the smart card and the card reader. The sizes of the fingerprint image and the features are $140 \mathrm{~KB}$ and about $1 \mathrm{~KB}$, respectively. As shown in Table 3, the time to require to encrypt and decrypt the fingerprint image using the AES algorithm are about 9.7 seconds and 13.8 seconds in the ARM7TDMI core, respectively. By comparison, the features require only 0.06 second (encryption) and 0.1 second (decryption). Also, as shown in Table 4, SHA1, and ECC for the digital signature can be executed in real-time for the fingerprint image and the features. If a core of the smart card is the 8051 chip, it is impossible to execute the AES algorithm for the fingerprint image in real-time. Furthermore, the digital signature cannot be executed in real-time because the time to require for the ECC algorithm is about 8 seconds.

Finally, the following configuration is assumed to evaluate the performance of each scenario of the client-server model (see Section 4). First, the client and the server have Pentium $4(2 \mathrm{GHz})$ processor and Xeon $(3 \mathrm{GHz})$ processor, respectively. The transmission speed of the Internet is assumed to be $10 \mathrm{Mbps}$. 128-bit AES, 1024-bit ECC, and SHA1 $[15,16]$ are used as symmetric encryption algorithm, digital signature algorithm, and hash algorithm, respectively, in order to guarantee both the integrity and the confidentiality of the transmitted information. Also, we examined each scenario on the fingerprint images captured with an 
TABLE 4: Number of instructions and cycles required for the digital signature.

\begin{tabular}{|c|c|c|c|c|c|}
\hline & & \multicolumn{2}{|c|}{ SHA1 } & \multicolumn{2}{|c|}{ ECC (1024-bit) } \\
\hline & & No. of instruction & No. of cycle & No. of instruction & No. of cycle \\
\hline \multirow{2}{*}{ ARM7TDMI } & $140 \mathrm{~KB}$ & 3091169 & 4709469 & 12528848 & 20327978 \\
\hline & $1 \mathrm{~KB}$ & 26990 & 42165 & 12528848 & 20327978 \\
\hline
\end{tabular}

TABLE 5: Evaluation data of cryptography algorithms (Byte, milisecond).

\begin{tabular}{lcccccc}
\hline & & Generate signature & Verify signature & Size of signature & AES encrypt & AES decrypt \\
\hline \multirow{2}{*}{ Pentium 4} & $1 \mathrm{~KB}$ & 6.359 & 33.656 & 48 & 3.0 & 4.0 \\
& $140 \mathrm{~KB}$ & 74.703 & 98.922 & 47 & 234.0 & 328.0 \\
\hline \multirow{2}{*}{ Xeon } & $1 \mathrm{~KB}$ & 3.656 & 19.797 & 48 & 1.0 & 2.0 \\
& $140 \mathrm{~KB}$ & 42.844 & 59.046 & 47 & 125.0 & 218.0 \\
\hline
\end{tabular}

optical scanner manufactured by NitGen [32]. The size of the fingerprint image and the fingerprint feature is about 140 KByte and 1 KByte, respectively. Finally, the disk access time of the fingerprint data stored in the server is assumed to be 50 miliseconds.

Table 5 shows the measured execution time of several cryptography algorithms used in our evaluation. These data are measured by arithmetic mean of 1000 executions on each platform.

5.2. Performance Evaluation Results for the Smart CardReader Model. As explained earlier, in the case of the smart card with the 8051 chip, secure transmission of both the fingerprint image and the features for all scenarios cannot be guaranteed because ECDSA, especially the ECC and the SHA1 algorithms, cannot be executed in real time.

When the smart card employs the ARM7TDMI core [29], the performance of each of five scenarios is evaluated as follows.

In SCENARIO 1, the cryptographic processes for guaranteeing the integrity and confidentiality of the sensitive fingerprint data transmitted between the smart card and the card reader can be executed in real-time because only the template stored in the smart card is transferred. It is, however, the Store-on-Card strategy that all the fingerprint verification modules except the storage module are executed in the card reader. Therefore, the fingerprint template stored in the smart card needs to be insecurely released into an external card reader in order to be compared with an input fingerprint.

SCENARIO 2 has the lowest security level because it is also the Store-on-Card strategies that the smart card only stores the fingerprint template. Furthermore, in SCENARIO 2 , secure transmission of the fingerprint image captured by the fingerprint sensor within the smart card cannot be guaranteed since the fingerprint sensor is built into the smart card. In this case, ECDSA, especially the ECC and the SHA1 algorithms, cannot be executed in real-time.

On the other hand, SCENARIO 3 is the most proper one to integrate fingerprint verification with the smart card because it guarantees higher security level than with the Store-on-Card. It also executes the cryptographic modules for secure transmission in real time because of transferring only the fingerprint features extracted in the card reader.

SCENARIO 4, like SCENARIO 2 cannot guarantee the security and real-time transmission of the fingerprint image captured by the fingerprint sensor within the smart card.

SCENARIO 5 is the System-on-Card that all the fingerprint verification modules take place on the card. This scenario is the best in terms of security as everything takes place on the smart card. As explained in Section 2.3, it is expensive and presents more than one realization problem. Even if the smart card employs the ARM7TDMI core, SCENARIO 5 cannot be executed in real-time because the feature extraction module of fingerprint verification is time consuming as shown in Table 2.

\subsection{Performance Evaluation Results for the Client-Server} Model. For each of the fingerprint authentication scenarios described in the previous section, we assume that the response time must be less than 5 seconds for real-time execution. As we expect, the server processes most of the time-consuming tasks in SCENARIO 1_1, whereas the clients have the heavy workload in SCENARIO 1_2. The extreme case is SCENARIO 1_3 where the clients do almost everything. However, the security of SCENARIO 1_3 is weaker.

In this section, we will evaluate the three scenarios in terms of the response time versus workloads imposed on the server. In other words, we will investigate the maximum workload that the server can handle with less than 5 seconds response time, or system time in queueing theory of each scenario. We adopt $\mathrm{M} / \mathrm{D} / 1$ queueing results assuming that the clients request services to the server in random fashion, but the server processes the jobs in deterministic fashion.

In an $\mathrm{M} / \mathrm{D} / 1$ system, the response time is given by

$$
w=\frac{1}{\mu}+\frac{\lambda}{2 \mu(\mu-\lambda)},
$$

where $W$ is the response time (or the system time), $\mu$ is the service rate, and $\lambda$ is the arrival rate. Here, $\lambda$ is the job request rate to the server by the clients, and $\lambda$ is assumed to increase as the number of clients increases. 
In SCENARIO 1_1, fingerprint acquiring (1000 milliseconds) is done by the sensor, whereas feature extraction (225 milliseconds), generation of signature for feature (6.359 milliseconds), and encryption (3.0 milliseconds) tasks are done by the client. Additionally, we consider the communication setup time for sending and receiving data from the server as st and the data transmission time to be 1.6 milliseconds: that is, $2 \times 0.8$ milliseconds or 2 times of $1 \mathrm{~KB}$ by $10 \mathrm{Mbps}$ Internet transmission. Thus, the total sums to $1235.959+5$ st milliseconds. We take values for st of 1 , 5 , or 50 millisecond(s) depending on the communication environments. On the server side, decryption for live feature (2.0 milliseconds), decryption for smart card feature (2.0 milliseconds), verifying the signs for live feature and smart card feature $(2 \times 19.797$ milliseconds), matching twice ( 2 $\times 6.5$ milliseconds), and data transmission time of 1.6 milliseconds and the communication setup time ( $5 \mathrm{st}$ ) sum to $58.194+5$ st, which equal the service time $(1 / \mu)$. Thus, we may build the response time $(W)$ in the $\mathrm{M} / \mathrm{D} / 1$ system as

$$
w=1235.959+5 \mathrm{st}+\frac{1}{\mu}+\frac{\lambda}{2 \mu(\mu-\lambda)}<5000 .
$$

If we take st be 1 millisecond in (2), we have $\lambda$ being less than 0.01569 in order to meet the total response time being less than 5 seconds. Here, the workload can be interpreted as the number of job requests by the clients to the server in unit time (millisecond).

A similar approach can be applied to SCENARIO 1_2: fingerprint acquiring ( 1000 milliseconds) is done by the sensor, whereas live feature extraction (225 milliseconds), decryption for smart card feature (4.0 milliseconds), verifying sign for smart card feature (33.656 milliseconds), and matching smart card versus live feature (10 milliseconds) tasks are done by the client. Additionally, we consider the communication setup time for sending and receiving data from the server as st and the data transmission time $(0.8$ millisecond, 1 time of $1 \mathrm{~KB}$ by $10 \mathrm{Mbps}$ Internet transmission). Thus, the total sums to $1273.456+4$ st milliseconds. We take values for st of 1,5 , or 50 millisecond(s) depending on the communication environments. On the server side, decryption for smart card feature (2.0 milliseconds), verify the sign for smart card feature (19.797 milliseconds), matching once (6.5 milliseconds) and data transmission time of 0.8 millisecond, and the communication setup time (4 st) sum to $29.097+4$ st, which plays the service time $(1 / \mu)$. Thus, we may build the response time $(W)$ in the M/D/1 system as

$$
w=1273.456+4 s t+\frac{1}{\mu}+\frac{\lambda}{2 \mu(\mu-\lambda)}<5000 .
$$

If we take st to be 1 millisecond in (3), $\mu$ must be less than 0.03008 in order to meet the total response time being less than 5 seconds.

In SCENARIO 1_3, the server is doing practically nothing except encrypting the stored template and sending the encrypted data to the clients. Fingerprint acquiring (1000 milliseconds) is done by the sensor, whereas decryption for DB feature (4.0 milliseconds), verifying signature for DB
TABLE 6: Summary of performance evaluation 1 ( $\mathrm{st}=1 \mathrm{~ms})$.

\begin{tabular}{lccc}
\hline SCENARIO & $1 \_1$ & $1 \_2$ & $1 \_3$ \\
\hline Maximum workload & 0.01569 & 0.03008 & 0.15010 \\
Relative workload & 1 & 1.92 & 9.57 \\
\hline
\end{tabular}

TABLe 7: Summary of performance evaluation 2 (st $=5$ milliseconds).

\begin{tabular}{lccc}
\hline SCENARIO & $1 \_1$ & $1 \_2$ & $1 \_3$ \\
\hline Maximum workload & 0.01188 & 0.02023 & 0.06810 \\
Relative workload & 1 & 1.70 & 5.73 \\
\hline
\end{tabular}

TABLE 8: Summary of performance evaluation 3 (st $=50$ milliseconds).

\begin{tabular}{lccc}
\hline SCENARIO & $1 \_1$ & $1 \_2$ & $1 \_3$ \\
\hline Maximum workload & 0.00310 & 0.00422 & 0.00942 \\
Relative workload & 1 & 1.36 & 3.04 \\
\hline
\end{tabular}

feature (33.656 milliseconds), decryption for smart card feature (4.0 milliseconds), verifying sign for smart card feature (33.656 milliseconds), live feature extraction (225 milliseconds) and 2 times of matching $(2 \times 10$ milliseconds $)$ tasks are done by the client. Additionally, we consider the communication setup time for sending and receiving signals from the server as st. Thus, the total sums to $1320.312+2$ st milliseconds. We take values for st of 1 , 5 , or 50 millisecond(s) depending on the communication environments. On the server side, encryption for DB feature (1.0 millisecond), generating signature for DB feature (3.656 millisecond(s)), and the communication setup time ( $2 \mathrm{st})$ sum to $4.656+2$ st, which plays the service time $(1 / \mu)$. Thus, we may build the response time $(W)$ in the $\mathrm{M} / \mathrm{D} / 1$ system as

$$
w=1320.312+2 \mathrm{st}+\frac{1}{\mu}+\frac{\lambda}{2 \mu(\mu-\lambda)}<5000 .
$$

Fixing st be 1 millisecond in (4), we have $\lambda$ being less than 0.1501 in order to meet the total response time being less than 5 seconds.

Summarizing the results obtained in this section is depicted in Tables 6-8. As we see in Table 6, the server can handle the lowest level of workload with SCENARIO 1_1, whereas the server can handle 9.57 times heavier workload with SCENARIO 1_3. In most reasonable case of SCENARIO 1_2, the server can handle 1.92 times heavier workload compared to the case of SCENARIO 1_1.

Another interesting result comes when we vary the communication setup time from $1 \mathrm{~ms}$ to $5 \mathrm{~ms}$ and $50 \mathrm{~ms}$, as shown in Tables 7 and 8 . As we notice in Tables 7 and 8 , the workload that the server can handle within the specified time constraint of 5 seconds changes dramatically as the communication setup time increases. When the communication setup time becomes 5 milliseconds, the server can handle only 5.73 times heavier workload with SCENARIO 1_3 compared to those with SCENARIO 1_1. It becomes worse with the communication setup time being 50 milliseconds, where the server can handle only 3.04 
times heavier workload with SCENARIO 1_3 compared to those with SCENARIO 1_1 even though almost everything is performed in the clients with SCENARIO 1_3.

\section{Conclusions}

We focus on examining which strategies are most appropriate to guarantee the privacy as well as the real time execution requirements for smart card with client-server based fingerprint verification in healthcare information systems. First, five scenarios with three different strategies for integrating fingerprints into a smart card system were examined. Then, three scenarios for implementing of the fingerprint authentication service for large-scale applications were examined with increasing numbers of clients.

Typical task assignment scenarios for fingerprint verification were considered to protect the fingerprint information transmitted and to guarantee both the integrity and the confidentiality of the fingerprint data. The workload characteristics of fingerprint verification and the authentication protocol were obtained by measuring the performance of the primitive operations on an ARM7TDMI-based smart card, a Pentium4-based PC, and a Xeon-based server, respectively. Based on these measured performance, the workload of each scenario of the task assignment was computed and was applied to the smart card reader model. Then, the collective performance of each scenario was analyzed using the workload computed for each scenario.

The analysis results showed that the scenario where the match operation is performed on the smart card with the fingerprint sensor being built into the card reader is the most beneficial in the smart card-card reader model. For large-scale applications, however, this scenario may not be applicable due to the high implementation cost. In the client-server model, the server could handle the lowest level of workload when it does decryption, verification and matching. By comparison, the server can handle 9.57 times heavier workload when the clients do decryption, verification, and matching.

\section{Acknowledgments}

This research was supported by The Ministry of Knowledge Economy (MKE), South Korea, under the Home Network Research Center (HNRC) Information Technology Research Center (ITRC) support program supervised by the National IT Industry Promotion Agency (NIPA), (NIPA -2009-C10900902-0035).

\section{References}

[1] H. Dreifus and T. Monk, Smart Cards, John Wiley \& Sons, New York, NY, USA, 1997.

[2] A. Alkhateeb, T. Takahashi, S. Mandil, and Y. Sekita, "The changing role of health care IC card systems," Computer Methods \& Programs in Biomedicine, vol. 60, no. 2, pp. 83-92, 1999.

[3] G. Kardas and E. T. Tunali, "Design and implementation of a smart card based healthcare information system," Computer
Methods \& Programs in Biomedicine, vol. 81, no. 1, pp. 66-78, 2006.

[4] M. Marschollek and E. Demirbilek, "Providing longitudinal health care information with the new German Health Card-a pilot system to track patient pathways," Computer Methods \& Programs in Biomedicine, vol. 81, no. 3, pp. 266-271, 2006.

[5] Gemalto, http://www.gemalto.com/.

[6] Health Smart Card, http://www.healthsmartcard.net/.

[7] C. Pagetti, et al., "A European health card," Final Report, European Parliament, Directorate General for Research, Document for STOA Panel, 2001.

[8] Transcards Project, http://www.sesamvitale.fr/programme/ programme_eng.asp.

[9] Netlink Project, http://www.sesam-vitale.fr/netlink/netlk_pres .htm.

[10] Netcards, Trans-European Healthcare Facility Service for Mobile Citizens, http://netcards-project.com/web/frontpage.

[11] G. Kardas and E. T. Tunali, "Design and implementation of a smart card based healthcare information system," Computer Methods \& Programs in Biomedicine, vol. 81, no. 1, pp. 66-78, 2006.

[12] O. Rienhoff, Integrated Circuit Health Data Cards (Smart Cards): A Primer for Health Professionals, PAHO, Washington, DC, USA, 2003.

[13] Health Insurance Portability and Accountability Act of 1996 (U.S.), Public Law no. 104-191, 110 Stat. 1936, HIPAA.

[14] B. Barber, "Patient data and security: an overview," International Journal of Medical Informatics, vol. 49, no. 1, pp. 19-30, 1998.

[15] W. Stallings, Cryptography and Network Security: Principles and Practice, Prentice-Hall, Upper Saddle River, NJ, USA, 2003.

[16] D. Hankerson, A. Menezes, and S. Vanstone, Guide to Elliptic Curve Cryptography, Springer, New York, NY, USA, 2003.

[17] S. Nanavati, M. Thieme, and R. Nanavati, Biometrics: Identity Verification in a Networked World, John Wiley \& Sons, New York, NY, USA, 2002.

[18] D. Maltoni, D. Maio, A. Jain, and S. Prabhakar, Handbook of Fingerprint Recognition, Springer, New York, NY, USA, 2003.

[19] R. M. Bolle, J. H. Connell, and N. K. Ratha, "Biometric perils and patches," Pattern Recognition, vol. 35, no. 12, pp. 27272738, 2002.

[20] B. Schneier, "The uses and abuses of biometrics," Communications of the ACM, vol. 42, no. 8, p. 136, 1999.

[21] S. Prabhakar, S. Pankanti, and A. K. Jain, "Biometric recognition: security and privacy concerns," IEEE Security and Privacy, vol. 1, no. 2, pp. 33-42, 2003.

[22] S. de Lusignan, T. Chan, A. Theadom, and N. Dhoul, "The roles of policy and professionalism in the protection of processed clinical data: a literature review," International Journal of Medical Informatics, vol. 76, no. 4, pp. 261-268, 2007.

[23] Y. S. Moon, H. C. Ho, K. L. Ng, S. F. Wan, and S. T. Wong, "Collaborative fingerprint authentication by smart card and a trusted host," in Proceedings of the Canadian Conference on Electrical and Computer Engineering, vol. 1, pp. 108-112, 2000.

[24] L. Rila and C. Mitchell, "Security analysis of smartcard to card reader communications for biometric cardholder authentication," in Proceedings of the 5th Smart Card Research and Advanced Application Conference (CARDIS '02), pp. 1928, 2002.

[25] D. Moon, et al., "Performance analysis of the Match-on-Card system for the fingerprint verification," in Proceedings of the 
International Workshop on Information Security Applications, pp. 449-459, 2001.

[26] Y. Chung, D. Moon, T. Kim, and J.-W. Park, "Workload dispatch planning for real-time fingerprint authentication on a sensor-client-server model," in Proceedings of the 5th International Conference on Parallel and Distributed Computing: Applications and Technologies (PDCAT '04), vol. 3320 of Lecture Notes in Computer Science, pp. 833-838, 2004.

[27] X.9.84, http://www.x9.org/home.

[28] A. D. Boyd, C. Hosner, D. A. Hunscher, B. D. Athey, D. J. Clauw, and L. A. Green, 'An 'Honest Broker' mechanism to maintain privacy for patient care and academic medical research," International Journal of Medical Informatics, vol. 76, no. 5-6, pp. 407-411, 2007.

[29] C. Quantin, O. Cohen, B. Riandey, and F.-A. Allaert, "Unique Patient Concept: a key choice for European epidemiology," International Journal of Medical Informatics, vol. 76, no. 5-6, pp. 419-426, 2007.

[30] D. Burger and T. Austin, "The simplescalar tool set, version 2.0," Tech. Rep., University of Wisconsin, Madison, Wis, USA, 1997.

[31] ARM, http://www.arm.com/.

[32] Nitgen, http://www.nitgen.com/. 